\title{
Concomitance of diabetic neuropathic amyotrophy and cachexia: a case report with review of the literature
}

\author{
Tuba Tulay Koca \\ Department of Physical Medicine and Rehabilitation, Malatya State Hospital, Malatya, Turkey
}

\begin{abstract}
Diabetic amyotrophy is a rare condition in which patients develop severe aching or burning pain in hips and thighs. This is followed by weakness and wasting of the muscles of proximal lower extremities, which often occur asymmetrically. Diabetic neuropathic cachexia is a different type of diabetic neuropathies. It leads to intense pain in affected extremities accompained by anorexia, weight loss as well as mood and sleep disturbances. A 42-year-old type 2 diabetic woman with a known poor glycemic control presented with loss of appetite, and weight (approximately $15 \mathrm{~kg}$ ), severe burning sensation over her lower limbs, depression and sleep disturbances for 3 months. Symmetric wasting was noted in her proximal lower extremities with bilateral muscle weakness. Her patella and Achilles reflexes were absent with decrease in her upper extremity reflexes. We evaluated the patient as diabetic amyotrophy associated with diabetic neuropathic cachexia based on clinical signs, electrophysiological and radiological examination findings. Physicians should take into consideration these rare complications of diabetes mellitus showing characteristics different from other types of neuropathies.
\end{abstract}

Keywords: Amyotrophy; atrophy; cachexia, diabetic neuropathy.

$\mathrm{D}$ iabetic neuropathy (DN) is involvement of nerves secondary to diabetes, and affects more than $50 \%$ of the diabetics. Its frequently seen complaints are tingling, pain, numbness, and weakness of hands, and feet. DN can be divided into three types based on its clinical process, distribution, type, and size of the nerve fibers as follows: generalized symmetric (distal sensory, sensorimotor polyneuropathy, small fiber polyneuropathy, autonomic polyneuropathy, sensory neuropathy of large my- elinated fibers), focal, and asymmetric neuropathies (cranial neuropathy, thoracic neuropathy, mononeuropathies of extremities, proximal motor polyneuropathy), combined forms (polyradiculopathy, diabetic neuropathic cachexia). Most frequently, distal symmetric, and sensory-motor types are seen.

Diabetic amyotrophy (DA) is a rarely seen complication characterized frequently by loss of asymmetric proximal motor function, atrophy, sensation of severe burning, and aching pain involving lower

Received: December 13, 2014 Accepted: March 23, 2015 Online: September 25, 2015

Correspondence: Dr. Tuba Tulay KOCA. Malatya Devlet Hastanesi,

Fiziksel Tip ve Rehabilitasyon Klinigi, Malatya, Turkey.

Tel: +90 416 - 2282800 e-mail: tuba_baglan@yahoo.com

(c) Copyright 2015 by Istanbul Northern Anatolian Association of Public Hospitals - Available online at www.kuzeyklinikleri.com 

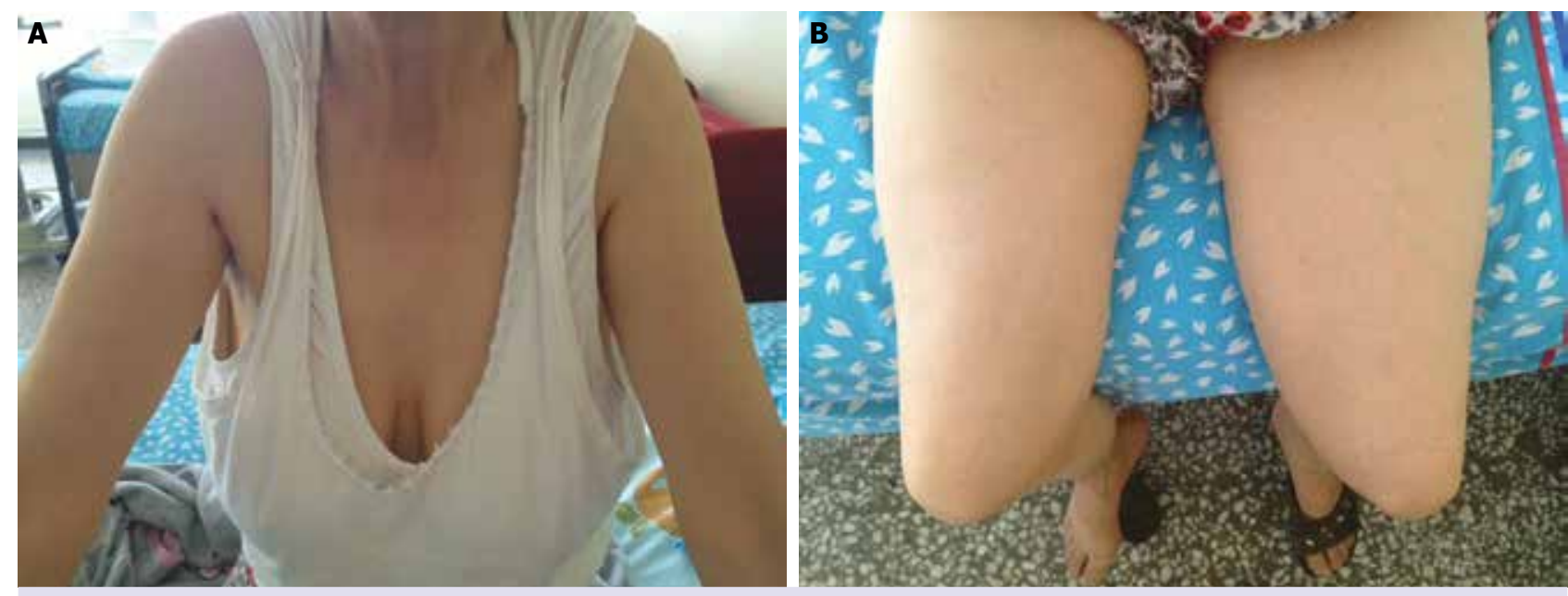

FIGURE 1. (A) Bilateral deltoid muscle atrophy is seen. (B) Bilateral quadriceps muscle atrophy is seen.

extremities. It is more frequently seen in male patients with type 2 diabetes older that 50 years of age. It is firstly defined by Bruns in the year 1980, and Garland used the term "diabetic amyotrophy". Nowadays, it is termed as 'Bruns-Garland syndrome' [1, 2, 3]. Dyck et al. [4] suggested the nomenculature 'diabetic lumbosacral radiculooplexopathy' for this disorder. In consideration of the anatomical distribution of the lesion it seems to be an accurate term. DA displays a clinical picture different from other types of neuropathy with its pathogenetic mechanism, and clinical manifestations. It may have an acute or subacute onset. Frequently, distal sensory neuropathy accompanies DN. Depression, and weight loss can be seen.

Diabetic neuropathic cachexia (DNC) is a rarely seen form of peripheral neuropathy. It is a complication characterized by weight loss, sleep disorders, and severe muscle wasting in patients with severe neuropathic complaints especially involving femoral regions bilaterally, and resolving spontaneously. DNC is generally seen in diabetic patients with poor glycemic control. Most of the patients are middle aged type 2 diabetics. Majority of them recover spontaneously, while permanent deficits can also develop [5].

Herein, we evaluated a 42-year-old type 2 diabetic female patient with acute weight loss, motor deficits in proximal muscles of lower extremities, and severe neoropathic pains diagnosed as diabetic proximal neuropathy (amyotrophy)' associated with 'diabetic neuropathic cachexia' based on clinical, electrophysiologic, and radiological examinations. We analyzed these two rarely seen complications in the light of the literature.

\section{CASE REPORT}

A 42-year-old female patient presented with complaints of severe pain in her left leg, muscle weakness, difficulty in climbing, and descending stairs, weight loss, loss of appetite, and sleep disorders with an onset dated back 3 months ago. Within the previous 3 months she had lost $15 \mathrm{~kg}$ (from $65 \mathrm{~kg}$ down to $50 \mathrm{~kg}$ ). She had type 2 diabetes for the last 15 years, and she was using antidiabetic drugs. Any past incident of systemic disease which might induce neuropathy and explain her generalized painful episodes apart from trauma, malignancy, and diabetes was not detected. Lumbar magnetic resonance imaging (MRI) obtained at L4-5 level revealed discal protrusion at midline. She indicated that she had received treatments for her lumbar hernia without any resolution of her severe pains. She was using gabapentin at three daily doses of $300 \mathrm{mg}$, and visual analogue scale (VAS) score of her pains was 9 points. Her body mass index (BMI) was $19.0 \mathrm{~kg} /$ $\mathrm{cm}^{2}(50 \mathrm{~kg} / 162 \mathrm{~cm})$.

On her physical examination an apparent muscle atrophy was detected on proximal muscles namely, deltoid, and quadriceps muscles of both upper, and lower extremities (Figures 1A, B). Motor deficit was not detected on upper extremity muscles, and she had hypoactive reflexes. Glove-stocking hypoes- 
thesia was present. Her hip movements were painful, and restricted. Shortened adductor, flexor, and hamstring muscle groups were detected.

Muscle strengths of the lower extremity were $3 / 5$ in the proximal, and $4 / 5$ in the distal muscle groups based on the evaluation of gross motor strengths. Bilateral Achilles, and patella reflexes could not be elicited Sensory examination was consistent with glove-stocking type hypoesthesia. Any pathological reflex was not detected.

Levels of fasting $(166 \mathrm{mg} / \mathrm{dl})$, and postprandial $(266 \mathrm{mg} / \mathrm{dl})$ blood sugar, and hemoglobin A1c (HbA1c: $7.8 \%$ (3-6\%) were as indicated. Whole blood cell counts, routine biochemical test results, and levels of acute phase reactants were within normal limits. Brucella agglutination test negativity was detected. While vitamin D, and B12, muscle enzyme levels, and thyroid function test results were within normal limits. Our patient's Beck Depression Inventory score was 28 points which complied with the clinical picture of moderate depression.

Patient's medical history revealed that she had poor glycemic control (HbA1c: 7.8\%), and she had not paid attention to her diet. Severe generalized pain all over her body, atrophy of proximal muscle groups of lower extremities, muscle weakness, and loss of some reflexes, diabetic amyotrophy and severe neuropathic pain suggested us the presence of diabetic amyotrophy, and associated distal sensory polyneuropathy. DNC also accompanied her complaints of insomnia, loss of appetite, serious weight loss (15 kg within the last 3 months), and depression. We performed electrophysiologic tests to reveal the presence of diabetic neuropathy.

During sensory nerve conduction studies, sensory nerve action potential of saphenous, and sural nerves could not be elicited. Distal latency of compound action potential of bilateral femoral nerves was prolonged (7.23-8.40 $\mathrm{msec})$. Results of bilateral median, and ulnar nerve motor conduction studies were within normal limits. Abnormal spontaneous activities (positive sharp wave, and fibrillation potentials) of bilateral iliopsoas, adductor magnus, and rectus femoris muscles were detected during needle EMG examinations. Muscle recruitment capability of the muscle, after full contraction decreased. The patient did not approve EMG examination of her lumbar paraspinal muscles.
These electrophysiologic findings strongly demonstrated involvement of femoral, obturator, peroneal, and tibial nerves, and provided supportive clinical evidence for the presence of diabetic lumbosacral plexopathy, and distal sensory polyneuropathy.

We offered a 3-week-long physiotherapy program to the patient which consisted of applications of heating pad, transcutaneous electrical stimulation (TENS), electrostimulation, stretching, joint range of movement (ROM), and strengthening exercises to relieve muscle weakness, and shortening. She was receiving gabapentin (300 $\mathrm{mg}$ tid) therapy. We added once daily doses of $600 \mathrm{mg}$ alpha lipoic acid and $25 \mathrm{mg}$ amitriptyline to her existing treatment regimen. At the end of 3 weeks, her pains regressed, and VAS scores dropped from 9 to 4 points. On control examination, hypoactive upper extremity reflexes were detected, while reflexes of the lower extremities could not be triggered. Muscle strengths did not change. She indicated improvement in her sleep patterns, and increase in her appetite. Our patient gain weight $(2 \mathrm{~kg})$ at the end of the 3 . week. Her control BMI was $19.8 \mathrm{~kg} / \mathrm{cm}^{2}$.

\section{DISCUSSION}

DA is a rare complication characterized by asymmetric weakness of proximal muscle groups of the lower extremities, muscle atrophy, and severe burning pain which can be seen in $0.8 \%$ of DM patients with subacute onset, and insufficient glycemic control. Although it is frequently seen in male patients over 50 years of age, it can be observed in youngsters, and female patients [6]. Typically it is unilateral, however bilateral involvements can be also seen. Our case differed from a classical DA clinic in that our patient was female younger than 50 years of age, and had asymmetric lower extremity involvement. Physiopathogenesis, and location of this condition which was termed as diabetic lumbosacral plexopathy are still debatable, and have not been elucidated yet. This condition appears to be immune -mediated vascular inflammatory radiculoplexopathy. Most authors think immune vasculopathy as an etiologic factor of DA. This assumption supports the use of inmunomodulator agents in the treatment of various types of neuropathy including DA. Rarely cervical radiculoplexopathy involving upper extremities is seen $[7,8,9]$. 
DA-related morbidities include mainly difficulties experienced during climbing, and descending stairs, and raising from a sitting position because of pain, proximal muscle atrophy, and loss of strength. Our patient was also complaining of difficulties in climbing, and descending stairs, and raising from a sitting position. These patients usually recover within a few months. EMG, and nerve conduction studies carry importance in establishing diagnosis. Positive sharp waves, and fibrillation potentials related to adductor muscles of the hips, and quadriceps femoris muscles can be seen on needle EMG. In the presence of distal symmetric polyneuropathy, sural nerve action potential is not seen frequently, however motor action potential amplitudes of peroneal, and tibial components decrease. During femoral nerve conduction studies asymmetric amplitudes, and on needle EMGs of paraspinal muscles fibrillation, and positive sharp waves can be seen $[10,11]$.

In the differential diagnosis of DA, amyotrophic lateral sclerosis, cauda equina syndrome, Guillain Barré syndrome, hypothyroid miyopathy syndrome, limb-girdle muscular dystrophy, lumbar spondylosis/listhesis, Meralgia paresthetica, mononeuritis multiplex, postpolio syndrome, radiation -related lumbosacral plexopathy should be considered.

DNC is a rarely seen form of peripheral neuropathy contrary to other neuropathies, and it is characterized by marked weight loss, painful dyskinesias affecting the trunk, and extremities and spontaneous resolution within a year. Loss of appetite, dramatic weight loss, and severe burning pain felt on extremities are its typical symptoms. Sleep disorders, and depression frequently accompany DNC. Its etiology is not known for sure. Although the underlying potential mechanism has not been understood fully, its etiologies include nerve regeneration, metabolic abnormalities, hemodynamic abnormalities in endoneurium, and immunologic reaction against insulin. In these patients following initiation of insulin therapy, and rapid achievement of normoglycemia, endoneural arteriovenous shunt decreases endoneural ischemia. Rapid improvement of metabolic control in diabetic patients or administration of higher doses of insulin may play a role in the pathophysiology of the disease. Our patient indicated that her mood was better 6 months before, and she hadn't had sleep disorders previously. She also stated that regulation of blood glucose levels was not successful despite frequent arrangements of drug therapies. We learned that she hadn't given insulin treatment for the. regulation of blood sugar, however she was using oral antidiabetics.

Diabetic patients can encounter peripheral nerve disorders within a large spectrum. Rarely seen complications have not been defined, and understood completely. 'Insulin neuritis' or 'treatment-related neuropathy' is a reversible disorder which is characterized by acute severe distal extremity pain, peripheral nerve damage, and autonomic dysfunction, and it becomes apparent because of rapid glycemic control. This clinical condition has been reported in both type I, and 2 diabetics, and in diabetic patients with poor glycemic control who were using insulin or oral antidiabetics. Pathogenesis of this condition, and associated painful episodes have not been sufficiently understood, and mechanisms involving endoneural ischemia, hypoglycemic microvascular neuronal damage, and regeneration of nerve fibers have been held responsible for this condition. Pain may be felt on various parts of the body including the trunk, abdomen or even all over body [12]. Since our patient did not use insulin, and had not distal extremity pain, we didn't predominantly think of insulin neuritis. However chronic inflammatory demyelinating polyradiculopathy is an immune-mediated, treatable complication progressing with diffuse motor loss. Painful episodes generally do not accopany this condition [13].

In patients with Type I diabetes, apart from complications developing within weeks after insulin therapy, rarely any other complication develops within few years after insuin therapy. In newly diagnosed type 1 diabetic patients, diabetic or metabolic cataract is also a rare complication. In individuals having personal predisposing factors to the development of cataract, following rapid glycemic control, development of diabetic cataract can be seen. Therefore young diabetic patients with newly diagnosed diabetes should be kept under control at least for the first few years [14].

In the literature, following rapid glycemic control, we encountered a patient with type 1 diabetes who developed severely painful acute motor-sensory, and autonomic neuropathy confined to the abdomen. A dramatic symptomatic improvement had been ob- 
served 4 months later in this patient (like ours) who was given analgesics. Painful neuropathies confined to the abdomen following treatment for diabetes have been very rarely reported in case reports [15]. While regulating glycemic level in diabetic patients, it will be appropriate to refrain from inducing dramatic, and sudden changes in glycemic levels.

DNC is characterized by burning pain, and allodynia involving lower extremities. This disease can develop both in type 1 , and 2 diabetic patients independent from the duration of the disease. Though depression, and impotence are frequently seen autonomic symptoms, other autonomic symptoms can be also observed. Typically it is a monophasic disease, however recurrent cases have been also reported. As is the case with insulin neuritis, this condition can be reversible weeks or months after appropriate glycemic control. In both disorders pain can be refractory to treatment despite use of multiple analgesic drugs [14]. We informed the patient about potentially recurrent nature of the disease, and we kept her under close clinical surveillance. Most prominently, concomitant presence of the symptoms of DNC, DA, and distal sensory neuropathy in our patient creates difficulties in making an accurate clinical diagnosis. At first glance, following a detailed medical history, and physical examination of our patient with presumely discogenic pain, we observed clinical signs, and symptoms of three different types of DN in our patient, and adjusted our treatment accordingly.

An effective treatment of DNC does not exist. For the management of depression, and neuropathy frequently, antidepressants, tramadol, topical capsaicine, clonidine, and gabapentin have been used. Besides nutritional status of the patient should be regulated [16]. Various pathogenetic mechanisms including excessive production of mitochondrial free radicals because of oxidative stress secondary to hyperglycemia, and also excessively glycated products, activation of aldose reductase polyol pathway, and plymerase enzymes, exchange of sodium-potassium ATPase pump function, various apoptotic processes triggered by endoplasmic reticulum secondary to hyperglycemia, impaired nerve perfusion, dyslipidemia, low-level inflammation, calcium dysequilibrium have been held responsible in the development of neuropathy. Treatments for $\mathrm{DN}$ are mostly targeted at pathophysiology of the disease.
Clarification of these mechanisms will contribute to the treatment [17].

In the treatment of DA, immunosuppressive drugs as intravenous human immunoglobulin (IVIg), cyclophosphamide, azathioprine, and methylprednisolone can be used. Though effectiveness of IVIg treatment has not been demonstrated in controlled studies, improvement in clinical symptoms as pain, and muscle strength is seen. Effectiveness of tricyclic antidepressants in diabetic neuropathy has been demonstrated in many studies. Their therapeutic efficacy in neuropathic pain are related to their inhibitory effects on noradrenalin reuptake, and blockage of sodium, and calcium channels. Besides, therapeutic efficacy of anticonvulsants as gabapentin, and pregabalin, and an antioxidant as alpha-lipoic acid has been demonstrated in patients with diabetic neuropathy [18]. Thus, we initiated therapy with once daily doses of $600 \mathrm{mg}$ alpha-lipoic acid, and $25 \mathrm{mg}$ amitriptyline. Then daily dose of gabapentin was raised form $300 \mathrm{mg}$ t1d to $600 \mathrm{mg}$ t1d. The patient benefited from this treatment, her appetite, and sleeping pattern improved.

We investigated evidence of malignancy in our patients because of her complaints of loss of appetite, severe weight loss, and generalized pain. Thoracal, and abdominal tomographic, and brain MRI examination findings were within normal limits. We didn't think presence of malignancy in our patient who was consulted to the clinic of internal medicine. Apart from DM, any other systemic disease which might cause neuropathy was not detected. Diagnoses of brucellosis, hypothyroidism, and polymyositis were ruled out based on clinical examination, and laboratory test results.

\section{Conclusion}

$\mathrm{DN}$ is a frequently seen complication developed in diabetic patients. This complication is divided into different subtypes based on clinical course, distribution, type, and size of the nerve fibers involved, and pathophysiologic mechanism DA is a rarely seen complication characterized by asymmetric weakness of proximal muscle groups of the lower extremities, muscle atrophy, and severe burning pain which can be seen in $0.8 \%$ of DM patients with subacute onset, and insufficient glycemic control. DNC is a rare disorder developed in patients with poor 
glycemic control characterized by acute painful peripheral neuropathy, and unexpectedly increased weight loss without muscle weakness. Loss of appetite, dramatic weight loss, and burning pain felt on extremities are typical manifestations. Sleep disorders, and depression frequently accompany the condition. Our case conveys importance in that these two DN types were seen in combination.

Conflict of Interest: Any conflict of interest was not declared between personal and/or legal entities and/or institutions.

Financial Disclosure: The budget of the research was met by Education, and Planning Board of Haydarpasa Exemplary Training and Research Hospital.

\section{REFERENCES}

1. Bruns L. Uberneuritsche lahmungen beim diabetes mellitus. Berl Klin Wochenschr 1890;27:1-15.

2. Garland H. Diabetic amyotrophy. Br Med J 1955;2:128790.

3. Asbury AK. Proximal diabetic neuropathy. Ann Neurol 1977;2:179-80.

4. Dyck PJ, Norell JE, Dyck PJ. Microvasculitis and ischemia in diabetic lumbosacral radiculoplexus neuropathy. Neurology 1999;53:2113-21.

5. Neal JM. Diabetic neuropathic cachexia: a rare manifestation of diabetic neuropathy. South Med J 2009;102:327-9.

6. Bhada SK, Sahay RK, Jyotsa VP, Agrawal JK. Diabetic Neuropathy: Current Consepts. J İndian Academy of Clin Med 2001;4:305-18.

7. Sander HW, Chokroverty S. Diabetic amyotrophy: current con- cepts. Semin Neurol 1996;16:173-8.

8. Williams IR, Mayer RF. Subacute proximal diabetic neuropathy. Neurology 1976;26:108-16.

9. Dyck PJ, Windebank AJ. Diabetic and nondiabetic lumbosacral radiculoplexus neuropathies: new insights into pathophysiology and treatment. Muscle Nerve 2002;25:477-91.

10. Tataroglu C, Bicerol B, Kiylioglu N, Ozkul A, Akyol A. Proximal femoral conductions in patients with lumbosacral radiculoplexus neuropathy. Clin Neurol Neurosurg 2007;109:654-60.

11. Subramony SH, Wilbourn AJ. Diabetic proximal neuropathy. Clinical and electromyographic studies. J Neurol Sci 1982;53:293-304.

12. Knopp M, Srikantha M, Rajabally YA. Insulin neuritis and diabetic cachectic neuropathy: a review. Curr Diabetes Rev 2013;9:267-74.

13. Knopp M, Rajabally YA. Common and less common peripheral nerve disorders associated with diabetes. Curr Diabetes Rev 2012;8:229-36.

14. Yuen KC, Day JL, Flannagan DW, Rayman G. Diabetic neuropathic cachexia and acute bilateral cataract formation following rapid glycaemic control in a newly diagnosed type 1 diabetic patient. Diabet Med 2001;18:854-7.

15. Takayama S, Takahashi Y, Osawa M, Iwamoto Y. Acute painful neuropathy restricted to the abdomen following rapid glycaemic control in type 2 diabetes. J Int Med Res 2004;32:558-62.

16. Naccache DD, Nseir WB, Herskovitz MZ, Khamaisi MH. Diabetic neuropathic cachexia: a case report. J Med Case Rep 2014;8:20.

17. Albers JW, Pop-Busui R. Diabetic neuropathy: mechanisms, emerging treatments, and subtypes. Curr Neurol Neurosci Rep 2014;14:473.

18. Max MB, Lynch SA, Muir J, Shoaf SE, Smoller B, Dubner R. Effects of desipramine, amitriptyline, and fluoxetine on pain in diabetic neuropathy. N Engl J Med 1992;326:1250-6. 